\title{
Distributed Network Utility Optimization in Wireless Sensor Networks Using Power Control ${ }^{*}$
}

\author{
George Tichogiorgos ${ }^{1}$, Kin K. Leung ${ }^{1}$, Archan Misra ${ }^{2}$ and Tom LaPorta ${ }^{3}$ \\ ${ }^{1}$ EEE Department, Imperial College, London SW7 2BT, U.K. \\ ${ }^{2}$ IBM Research, New York, U.S.A. \\ ${ }^{3}$ The Pennsylvania State University, University Park, PA, U.S.A.
}

Email:\{georgios.tichogiorgos06, kin.leung\}@imperial.ac.uk, archan@us.ibm.com,tlp@cse.psu.edu

\begin{abstract}
We extend the existing network utility maximization (NUM) framework for wired networks to wireless sensor networks by formulating it in order to take into account interference among radio links. We study the conditions under which the formulated problem is a feasible convex optimization problem. Under such conditions, a distributed algorithm is proposed to solve the problem optimally. Finally, we provide numerical results, based on computer simulations, to show the performance of the proposed algorithm and the rate of convergence of its solution.
\end{abstract}

Index Terms-Network Utility Maximization, Power Control, Rate Allocation, Wireless Sensor.

\section{INTRODUCTION}

$\mathrm{W}$ ireless Sensor Networks are considered as a new class of distributed systems that are being extensively used to sense critical information that require us to take some actions. One of the most important challenges in wireless sensor networks is the development of a self-optimization technique that would enable sensors to adjust their transmissions for optimal network performance.

This paper makes the following contributions to the problem of optimizing wireless sensor networks with respect to network resource allocation:

1. Extend the network utility maximization (NUM) framework used in wired networks with the main characteristic of the wireless medium, namely, interference among links;

2. Propose a new formulation for the resource allocation;

3. Propose a distributed algorithm that is shown to solve

\footnotetext{
* This research was sponsored by US Army Research Laboratory and the UK Ministry of Defence and was accomplished under Agreement Number W911NF-06-3-0001. The views and conclusions contained in this document are those of the authors and should not be interpreted as representing the official policies, either expressed or implied, of the US Army Research Laboratory, the U.S. Government, the UK Ministry of Defense, or the UK Government. The US and UK Governments are authorized to reproduce and distribute reprints for Government purposes notwithstanding any copyright notation hereon.
}

the resource allocation problem optimally.

The remainder of this paper is organized as follows: Section 2 is a brief literature review of the most relevant work in the area of NUM. Section 3 includes a presentation of the reformulated problem, based on Power Control, and a solution of the problem with a distributed algorithm that is shown to solve it optimally. In addition, we state the necessary and sufficient conditions needed for feasibility of the Power Control mechanism. Section 4 provides some numerical results concerning the performance of the algorithm and, finally, Section 5 concludes our results and outlines of our future work.

\section{RELATED WORK}

\section{A. Wired networks}

Evidently, the first work on Network Utility Maximization was published in [1], which proposes the following formulation:

$$
\begin{aligned}
& \max \sum_{r \in R} U_{r}\left(x_{r}\right) \\
& \text { s.t. } \quad A x \leq C \\
& x \geq 0
\end{aligned}
$$

where $\mathrm{r}, x_{r}$ and $U_{r}$ denote the source sensor, the data rate of source sensor $r$ and the utility of sensor $r$ when transmitting at rate $x_{r}$ respectively. Then, element $A_{j r}$ is 1 when resource $j$ lies on route $r$, and 0 otherwise. The authors propose an algorithm that enables the network nodes to determine the optimal way to share the link bandwidths among different traffic flows using the max-min fairness criterion. The solution is a set of differential equations, proved to be stable. The same problem was also solved in [2] following a different methodology based on Lagrange theory and two algorithms, both synchronous and asynchronous, are proposed. In [3] and [4], the authors use multi-level decomposition techniques to provide different optimization algorithms, each with a different 
trade-off among convergence speed, message overhead and distributed computation architecture. A systematic framework is also presented to decompose the optimization problems.

\section{B. Wireless Networks}

The first attempt for NUM in wireless networks was made in [5]. Its most important contribution is the proof that the logarithmic relation between capacity and transmission power is neither convex nor concave and the proposal of a centralised algorithm converging to the optimal. In [6] and [7], the NUM framework was converted into mission-centric, where utility functions depend on more than one flows and flows may have more than one sinks. Moreover, the notion of maximal clique is introduced, which is a set of links that mutually interfere with each other. In addition, a distributed algorithm is proposed for solving the problem using a scheduling mechanism to avoid the difficulties of considering interference between links.

To the best of our knowledge, existing work on the NUM has not actually taken into account the major characteristic of wireless networks, the interference among links. The key contribution of this work is to propose a NUM framework that indeed takes interference into account and makes use of power control to cope with interference.

\section{Network Utility OPtIMIZATION Using POWER CONTROL}

\section{A. Problem Formulation}

Consider a set of $M$ randomly deployed sensors that act as data sources and send traffic to other nodes in a multihop wireless network. Assume that $\Omega=\{1,2, \ldots, S\}$ is the set of all nodes in the network and that each traffic flow is generated by one of the $M$ sensors with only one node as its destination. A rate vector $\underline{r}=\left[r_{1}, r_{2}, \ldots, r_{M}\right]^{T}$ denotes the transmission rates for all the individual nodes. Moreover, assume that there exists a set $T=\{1,2, \ldots, L\}$ of links in the network. There is a path loss matrix $G$ of size $L \times L$ which depends on the physical characteristics of the link and whose element $G_{i j}$ is the path loss gain from the transmitter of link $i$ to the receiver of link $j$. Vector $\underline{p}=\left[p_{1}, p_{2}, \ldots, p_{L}\right]^{T}$ consists of all the individual transmission powers of the links. Furthermore, we use a vector $\underline{c}=\left[c_{1}, c_{2}, \ldots, c_{L}\right]^{T}$ to denote link capacities. Each traffic flow $i$ is characterized by a utility function, $U_{i}\left(r_{i}\right)$, a function of the transmission rate of the flow. Moreover, there is a cost function $V_{j}\left(p_{j}\right)$ for each link $j$, which depends on its transmission power, $p_{j}$, and represents the cost of using the limited power resources in the wireless sensor network.

We propose the following NUM formulation:

$$
\begin{gathered}
\text { Problem } P: \quad \max _{\underline{r}, \underline{p}} \sum_{i=1}^{M} U_{i}\left(r_{i}\right)-\sum_{j=1}^{L} V_{j}\left(p_{j}\right) \\
\text { s.t. } \quad \sum_{i \in Z(j)} r_{i} \leq c_{j} \\
\frac{G_{j j} \cdot p_{j}}{\sum_{\substack{k=1 \\
k \neq j}}^{L} G_{j k} \cdot p_{k}+n_{j}} \geq \gamma_{j}
\end{gathered}
$$

$\forall \operatorname{link}$ j

where $\gamma_{j}$ is the target signal-to-interference-plus-noise ratio (SINR) for link $j$ and $Z(j)$ represents the set of traffic flows passing through each link $j$.

According to its first constraint, the total traffic flow passing from each link $j$ should not exceed the link capacity, $c_{j}$. This is actually the same constraint as in the NUM framework for wired networks [1]. Even though it is difficult to know the exact capacity of a wireless link, it is possible to estimate it under some specific conditions based on the SINR at each node of the network. This is the contribution of the second constraint which is actually an expression of the power control problem. According to these constraints, we maximize the objective function so that the SINR is at least equal to a target value, which is a measure of the quality offered to the users of the network. In that way, we can capture the interference between links. As a result, if we know that the SINR will always be higher than a certain limit, one can be sure that the capacity of a link will always be above a specific value ( $c_{j}$ in our case). Hence, the combination of the two constraints can adequately capture the necessary characteristics of wireless links. 


\section{B. Solution of the Problem}

Readers not familiar with Convex Optimization Theory are advised to refer to [8]. To solve problem $\mathrm{P}$ using local search algorithms, we need to make some assumptions about the concavity of the objective function. Both constraints of Problem $P$ are linear and, therefore, convex. So, according to [8], three conditions must hold:

- functions $U_{i}\left(r_{i}\right), i=1, \ldots, M$, must be strictly concave, increasing and twice differentiable functions of $r_{i}$ for $i=1, \ldots, M$,

- functions $V_{j}\left(p_{j}\right), j=1, \ldots, L$, must be strictly convex, increasing and twice differentiable functions of $p_{j}$ for $j=1, \ldots, L$,

- variables $r_{i}$ take values in the range $I_{r}=\left[m_{r}, M_{r}\right]$, where $m_{r} \geq 0$ and $M_{r}<\infty$, and $p_{j}$ take values in the range $I_{p}=\left[m_{p}, M_{p}\right]$, where $m_{p} \geq 0$ and $M_{p}<\infty$.

We regard Problem $P$ as our primal problem. As we can see, both source rates $r_{i}$ and transmission powers $p_{j}$ are coupled by constraints 1 and 2, respectively. So, in order to solve the problem in a distributed way, we need to look at the problem from a different point of view in order to reveal its distributed nature. Hence, we solve the Dual Problem. We define the Lagrangian function $L$ of an optimization problem:

$$
\begin{array}{ccc} 
& \min f_{0}(x) \\
\text { s.t. } & f_{i}(x) \leq 0, & i=1, \ldots, m \\
& \mathrm{~h}_{i}(x)=0, & i=1, \ldots, p
\end{array}
$$

as a function $L: R^{n} \times R^{p} \rightarrow R$ of the form:

$$
L(x, \mu, \lambda)=f_{0}(x)+\sum_{i=1}^{m} \mu_{i} f_{i}\left(x_{i}\right)+\sum_{i=1}^{p} \lambda_{i} h_{i}\left(x_{i}\right)
$$

Note that the variables $\mu_{i}$ and $\lambda_{i}$ are called Lagrange Multipliers and are also the optimization variables of the Dual Problem. Please, refer to [8] for a complete description of the Lagrangian function and its properties. The objective function of the Dual Problem is

$$
\begin{aligned}
& l(\underline{\mu}, \underline{\lambda})=\max _{r_{i} \in I_{r}, p_{j} \in I_{p}} L(\underline{x}, \underline{p}, \underline{\mu}, \underline{\lambda}) \Leftrightarrow \\
& l(\underline{\mu}, \underline{\lambda})=\sum_{i=1}^{M} \max _{r_{i} \in I_{r}}\left[U_{i}\left(r_{i}\right)-r_{i} \cdot \lambda^{i}\right]-
\end{aligned}
$$

$-\sum_{j=1}^{L} \min _{p_{j} \in I_{p}}\left[V_{j}\left(p_{j}\right)-\mu_{j}\left(G_{j j} p_{j}-\gamma_{j} \sum_{\substack{k=1, k \neq j}}^{L} G_{j k} p_{k}-\gamma_{j} n_{j}\right)\right]+\sum_{i=1}^{L} \lambda_{j} c_{j}$

Based on (5) we could define two optimization problems. The first one is:

Problem $P_{1}^{i}: \quad \max _{r_{i} \in I_{r}} U_{i}\left(r_{i}\right)-r_{i} \cdot \lambda^{i}$

where

$$
\lambda^{i}=\sum_{j \in S(i)} \lambda_{j}
$$

Each source sensor $i$ can solve the above problem in order to determine the optimal traffic rate that it should transmit its data. The only piece of information that each source sensor needs is the aggregate cost of the links it is using, which does not produce significant message overhead. Also note that problem $P_{1}^{i}$ is actually the maximum benefit that sensor $i$ can achieve for that given aggregate price of links.

The second distributed problem is:

Problem $P_{2}^{j}$ :

$$
\min _{p_{j} \in I_{p}}\left[V_{j}\left(p_{j}\right)-\mu_{j} \cdot\left(G_{j j} p_{j}-\gamma_{j} \sum_{\substack{k=1, k \neq j}}^{L} G_{j k} p_{k}-\gamma_{j} n_{j}\right)\right]
$$

It is clear that Problem $P_{2}^{j}$ can be solved by each link $j$ independently. At each iteration, link $j$ should be aware of the value of the dual variable $\mu_{j}$. Note that the optimal value of problem $P_{2}^{j}$ is actually the minimum transmission cost that a link can have for the given price $\mu_{j}$. So, the objective function of the Dual Problem can be written in the form:

$$
l(\underline{\mu}, \underline{\lambda})=\sum_{i=1}^{M} P_{1}^{i}-\sum_{j=1}^{L} P_{2}^{j}+\sum_{j=1}^{L} \lambda_{j} c_{j}
$$

Then, the Dual Problem of the network is given by:

$$
\begin{array}{rrr}
\text { Dual Problem } D: & \min _{\underline{\mu}, \underline{\lambda}} l(\underline{\mu}, \underline{\lambda}) & \\
& \text { s.t. } & \underline{\lambda} \geq \underline{0} \\
& & \underline{\mu} \geq \underline{0}
\end{array}
$$

Under the assumptions made earlier, the Duality Gap is zero and the optimal prices of the dual values, which are also the Lagrange multipliers of the Primal Problem, exist and hence the solution of the Dual problem is also the solution of the Primal one. In order to solve it, we will use an iterative 
algorithm, the Gradient Projection Method described in [9]. Recall that the recursive formula for determining the value of a variable $x$ at time $t+1$ is:

$$
x(t+1)=[x(t)-\alpha \cdot \nabla F(x(t))]^{+}
$$

where $\alpha$ is a positive constant. In our case, we obtain the update equations for the shadow prices $\mu_{j}$ and $\lambda_{j}$ as follows:

$$
\mu_{j}(t+1)=\left[\mu_{j}(t)-\alpha_{1}\left(-G_{j j} p_{j}\left(\mu_{j}\right)+\gamma \sum_{k=1, k \neq j}^{L} G_{j k} p_{k}+\gamma n_{j}\right)\right]^{+}
$$

and

$$
\lambda_{j}(t+1)=\left[\lambda_{j}(t)-\alpha_{2}\left(c_{j}-\sum_{i \in Z(j)} r_{i}(\underline{\lambda})\right)\right]^{+}
$$

where $[x]^{+}=\max (x, 0)$.

Using the same procedure, we also obtain the optimal solutions for problems $P_{1}^{i}$ and $P_{2}^{j}$ as

$$
r_{i}^{*}=\left[U_{i}^{\prime}\left(\sum_{j \in S(i)} \lambda_{j}\right)^{-1}\right]_{m_{r}}^{M_{r}}
$$

and

$$
p_{j}^{*}=\left[V_{j}^{\prime}\left(\mu_{j} G_{j j}\right)^{-1}\right]_{m_{p}}^{M_{p}}
$$

respectively, where $[x]_{a}^{b}=\min (\max (x, a), b)$.

\section{Distributed Optimization Algorithm}

We propose the following distributed algorithm that can be used by each node in the network to reach the optimal performance. The algorithm consists of two sub-algorithms, one carried out by each source sensor $i$ and another one implemented by each link $j$, as shown in tables 1 and 2 .

\section{TABLE I: SOURCE SENSOR'S $i$ DISTRIBUTED ALGORITHM}

Each time slot $t=1,2, \ldots$ do:

1. Receive the aggregate cost $\lambda^{i}$, given by (5), for all the links that sensor $i$ is using for sending its traffic.

2. Calculate the new data rate for time $t+1$ using (14)

3. Send the new rate $r_{i}(t+1)$ to all the links that sensor $i$ is using

The message overhead of the algorithm does not seem to be significant. The aggregate price of each flow can be calculated by sending a packet from sink to source and each time it reaches an intermediate node, the link increases the cost header by its shadow price. Then, when the packet reaches the source the value of this header variable will be equal to the aggregate $\operatorname{cost} \lambda^{i}$.

TABLE II: LINK's $j$ DistRIBUTED ALgORITHM

Each time slot $t=1,2, \ldots$ do:

1. Receive the aggregate traffic rate that is passing through it using formula $r^{j}=\sum_{i \in Z(j)} r_{i}$.

2. Calculate the value of the transmission power price $\mu_{j}$ for time $t+1$ using (12).

3. Calculate the value of the rate price $\lambda_{j}$ for time $t+1$ using (13).

4. Use the new value $\mu_{j}(t+1)$ in order to calculate the optimal transmission power $p_{j}^{*}$, using (15).

4. Send the new price $\lambda_{j}(t+1)$ to all the sources that are using link $j$.

\section{Feasibility of the Algorithm}

Even though we have proposed an algorithm for solving the NUM problem, this does not mean that the problem has always a feasible solution and hence we need to provide some necessary and sufficient conditions for feasibility. Observe that the problem $\mathrm{P}$ can be viewed as two separable subproblems that are connected by the objective function. The first one is the rate allocation problem and the second is the power control problem. The rate allocation sub-problem is actually presented and solved in [1] and [2], and is proved to be feasible since the zero vector $\underline{0}$ is a feasible point of the problem. On the contrary, the power control sub-problem is not always feasible. However, Lemma 1 in [10] represents a necessary and sufficient condition that guarantees the existence of a feasible solution. Accordingly, the SINR targets $\gamma_{j}, j=1, \ldots, L$, are feasible if and only if $\rho<1$ where $\rho$ is the Perron-Frobenius eigenvalue of the matrix $Y \cdot H^{-1} \cdot E$, where 


$$
Y=\left[\begin{array}{cccccc}
\gamma_{j} & 0 & & \cdots & & 0 \\
0 & \gamma_{j} & & \cdots & & 0 \\
\vdots & \vdots & \ddots & & & \vdots \\
\vdots & \vdots & & \ddots & & \vdots \\
\vdots & \vdots & & & \gamma_{j} & 0 \\
0 & 0 & & \cdots & 0 & \gamma_{j}
\end{array}\right]
$$

Matrix $\mathrm{H}$ is defined as $H_{i j}=\left\{\begin{array}{ll}G_{i i}, & i=j \\ 0, & i \neq j\end{array}\right.$ and matrix $E$ is $E_{i j}=\left\{\begin{array}{ll}0, & i=j \\ G_{i j}, & i \neq j\end{array}\right.$.

\section{NUMERICAL RESULTS}

In this section, we present numerical results to show the performance of the proposed distributed algorithm. In particular, we have simulated the algorithm in a network consisting of 10 sensor nodes and a total of 9 links, as shown in Figure 1. Assume that three sensors, sensors 1, 2 and 3, in the network generate and send data flows to three other sensors, sensors 7,9 and 10, and that the routing matrix of these flows is known a priori and fixed. For illustration purposes, we have set the utility functions $U_{i}\left(r_{i}\right)=\log \left(r_{i}\right)$ and the cost functions $V_{j}\left(p_{j}\right)=\left(p_{j}\right)^{2}$. Note that $U_{i}\left(r_{i}\right)$ is a concave function of $r_{i}$ and $V_{j}\left(p_{j}\right)$ is a convex function of $p_{j}$, as required by the conditions mentioned earlier. Finally, the SINR target at each link is equal to $\gamma_{\text {target }}=10 \mathrm{~dB}$ and the capacity of each link is equal to $c^{*}=10 \mathrm{Mbps}$.

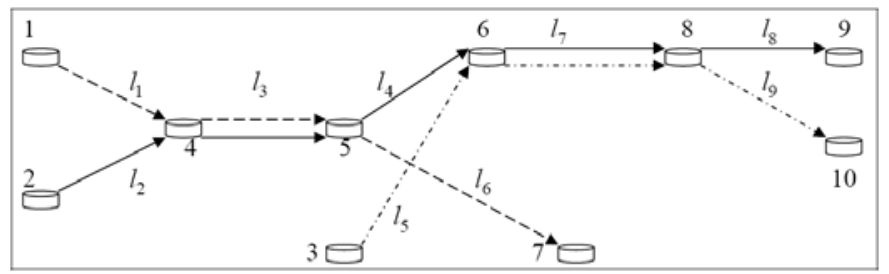

Figure 1 - The network topology

To study the convergence rate and the deviation from the optimality for the proposed algorithm, we have simulated our network for two different sets of update coefficients $\alpha_{1}$ and $\alpha_{2}$ and compared it with the actual optimal solution. The performance of our distributed algorithm is shown in Figures 2 to 6. According to [9], the values of $\alpha_{1}$ and $\alpha_{2}$ can be any positive and sufficiently small number, so that each iteration of the Gradient Projection method will decrease the value of the cost function unless we reach the optimal solution.

We show the convergence of the rate allocation for two flows (Figures 2 and 3) and transmission power of three links (Figures 4 and 5). Figure 6 depicts the convergence of the aggregate SINR error with respect to the SINR target. The convergence metric is a normalized summation of the differences between the actual and the target SINR values as follows:

$$
S I N R_{\text {perf }}=\frac{\sum_{i=1}^{M}\left|S I N R_{i}-\operatorname{SINR}_{\mathrm{target}}\right|}{S I N R_{\mathrm{target}}}
$$

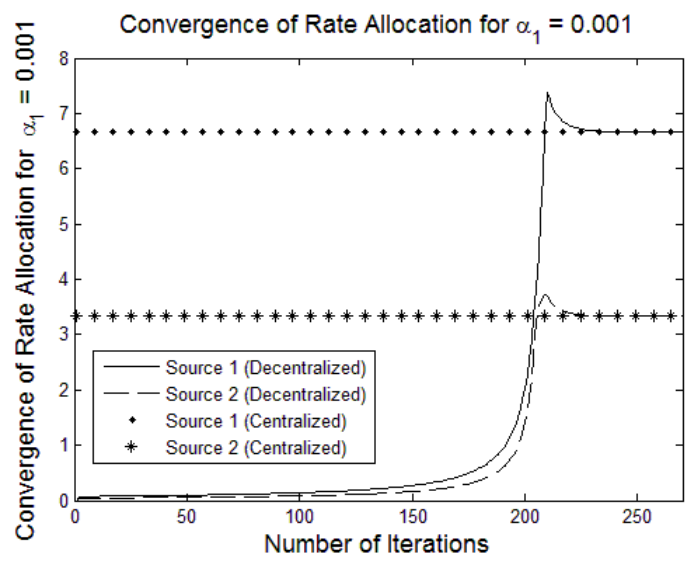

Figure 2 - Allocated Data Rate for $\alpha_{1}=0.001$

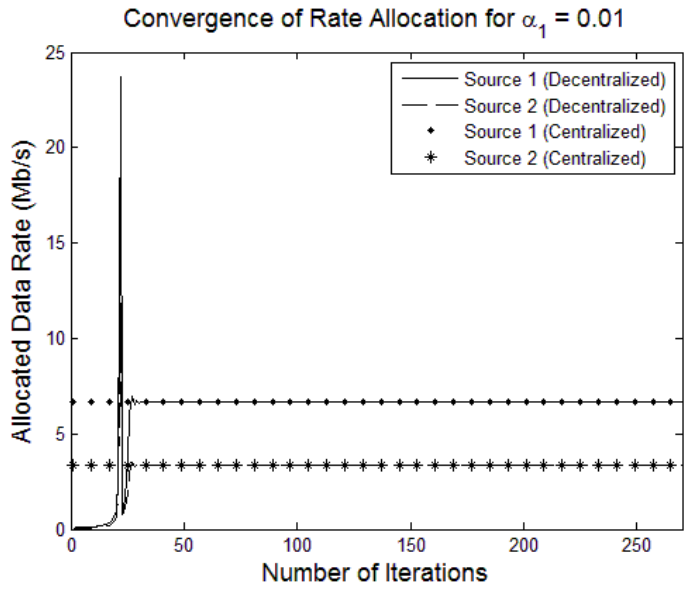

Figure 3 - Allocated Data Rate for $\alpha_{1}=0.01$

According to these graphs, it can be seen that for greater values of $\alpha_{1}$ and $\alpha_{2}$ the algorithm is converging quicker. However, the smaller the values of these parameters of the gradient projection method, the smoother the convergence to the optimal value will be. As shown in Figures 2 and 3, the allocated data rate may exceed the link capacity temporarily 
before it reaches the optimal solution at steady state. We further observe that the greater the coefficient $\alpha_{1}$, the greater the data rate may exceed the capacity temporarily. Therefore, it may be worthwhile to choose small coefficient values to limit such excessive data rate.

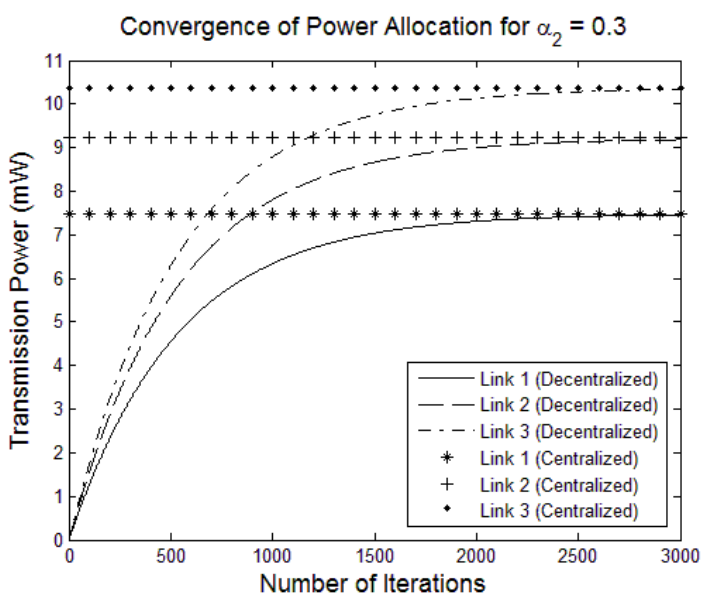

Figure 4 - Transmission Power for $\alpha_{2}=0.3$

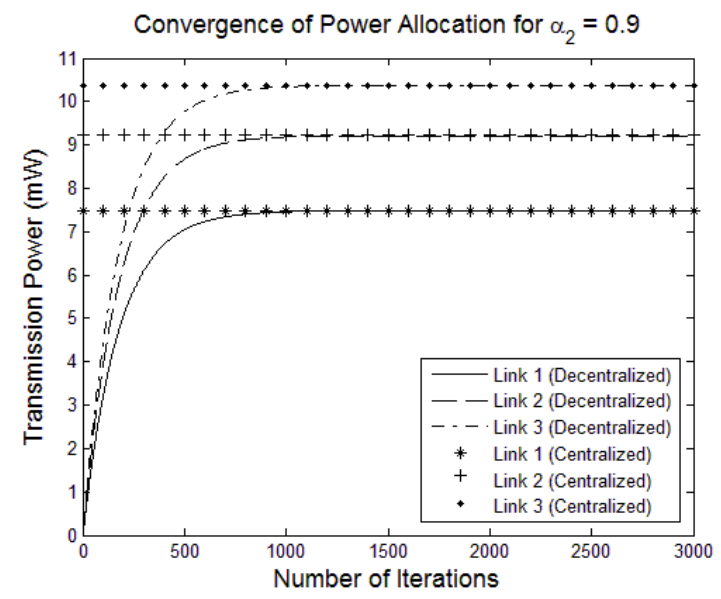

Figure 5 - Transmission Power for $\alpha_{2}=0.9$

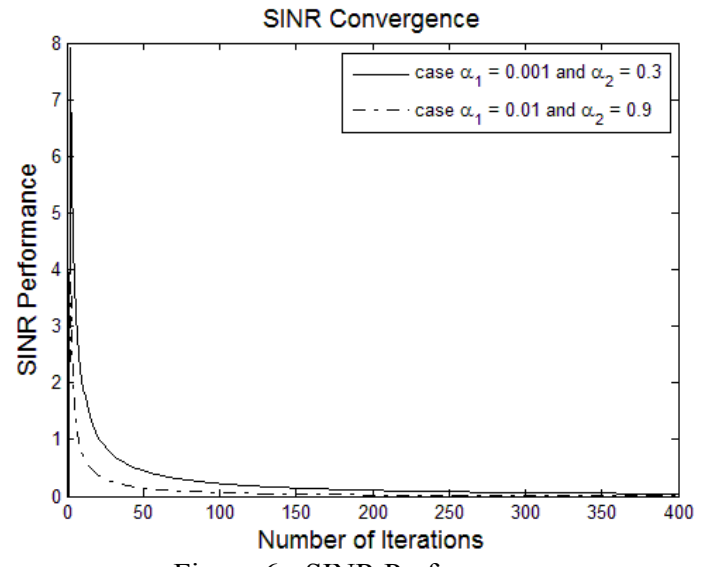

Figure 6 - SINR Performance
Hence, we see that the optimal decision for these parameters actually represents a trade-off between convergence rate and convergence smoothness in practice. Nevertheless, these simulation results reveal that the proposed algorithm always converges at the actual optimal value which means that it can indeed solve the maximization problem optimally by selecting appropriate data rates and transmission power levels.

\section{CONCLUSIONS AND FUTURE WORK}

In this paper, we have extended the existing Network Utility Maximization framework for wireless networks so that it takes into account the interference between links and the path loss effect by proposing a problem formulation based on power control. In addition, we have proposed a distributed algorithm for the optimization problem and obtained the conditions under which the problem is solvable and a feasible solution exists. Finally, we have verified the performance of the proposed algorithm in terms of convergence of rate allocation and transmission power by simulation.

We plan to extend the proposed framework further so that it takes into account multi-sink and multi-source flows. In addition, we also prefer to enhance the formulation to capture the coupling between SINR and link capacity.

\section{REFERENCES}

[1] F. P. Kelly, A. K. Maulloo, D. K. H. Tan, "Rate Control for Communication Networks: Shadow Prices, Proportional Fairness and Stability”, Journal of the Operational Research Society, Vol. 49, 1998, pp. 237-252.

[2] S. H. Low and D. E. Lapsey, "Optimization Flow Control, I: Basic Algorithm and Convergence", IEEE/ACM Transactions on Networking, Vol. 7, Dec. 1999, pp. 861-87

[3] D. Palomar and M. Chiang, "Alternative Distributed Algorithms for Network Utility Maximization: Framework and Applications", To appear in IEEE Transactions on Automatic Control, March 2008

[4] D. Palomar and M. Chiang, "A Tutorial on Decomposition Methods for Network Utility Maximization", IEEE Journal on Selected Areas in Communications, Vol. 24, No. 8, August 2006

[5] M. Chiang, "Balancing Supply and Demand of Bandwidth in Wireless Cellular Networks: Utility Maximization over Powers and Rates", Proc. IEEE INFOCOM, Hong Kong, China, March 2004

[6] S. Eswaran, A. Misra and T. LaPorta, "Distributed Utility Optimization in Mission-Oriented Wireless Sensor Networks", Annual Conference of ITA, September 2007.

[7] S. Eswaran, A. Misra and T. LaPorta, "Distributed Utility Optimization in Mission-Oriented Wireless Sensor Networks", submitted for publication at IEEE INFOCOM 2008.

[8] S. Boyd and L. Vandenberghe, Convex Optimization, Cambridge University Press, $1^{\text {st }}$ edition, 2004

[9] D. P. Betsekas and J. N. Tsitsiklis, Parallel and Distributed Computation: Numerical Methods, $1^{\text {st }}$ Edition, Athena Scientific, Belmont, Massachusetts, 1997.

[10] S. Ulukus and R. D. Yates, "Stochastic Power Control for Cellular Radio Systems", IEEE Trans. on Communications, Vol. 45, No. 6, June 1998. 